\title{
BMJ Open Protocol for a systematic literature review and network meta-analysis of the clinical benefit of inhaled maintenance therapies in chronic obstructive pulmonary disease
}

\author{
Adam Lewis, ${ }^{1}$ Eleanor L Axson, ${ }^{\oplus 1}$ James Potts, ${ }^{1}$ Renelle Tarnowska, ${ }^{2}$ \\ Helene Vioix, ${ }^{2}$ Jennifer K Quint ${ }^{\oplus 3}$
}

To cite: Lewis A, Axson EL, Potts J, et al. Protocol for a systematic literature review and network meta-analysis of the clinical benefit of inhaled maintenance therapies in chronic obstructive pulmonary disease. BMJ Open 2019;9:e025048. doi:10.1136/ bmjopen-2018-025048

\section{- Prepublication history for} this paper is available online. To view these files, please visit the journal online (http://dx.doi. org/10.1136/bmjopen-2018025048).

Received 27 June 2018 Revised 6 November 2018 Accepted 29 January 2019

D) Check for updates

C Author(s) (or their employer(s)) 2019. Re-use permitted under CC BY-NC. No commercial re-use. See rights and permissions. Published by BMJ.

${ }^{1}$ Department of Respiratory Epidemiology, National Heart and Lung Institute, Imperial College London, London, UK ${ }^{2}$ Boehringer Ingelheim Ltd, Bracknell, UK

${ }^{3}$ Department of Respiratory Epidemiology, Occupational Medicine and Public Health, Imperial College London, London, UK

Correspondence to

Dr Adam Lewis;

adam.lewis@imperial.ac.uk

\section{ABSTRACT}

Introduction Chronic obstructive pulmonary disease (COPD) exacerbations progress the course of disease and impair lung function. Inhaled maintenance therapy reduces exacerbations. It is not yet established which inhaled therapy combination is best to reduce exacerbations, lung function decline and symptom burden.

Methods and analysis MEDLINE, EMBASE and the Cochrane Library will be searched for articles between January 2011 and May 2018 using a pre-specified search strategy. Conference proceedings will be searched. Systematic reviews (with or without meta-analysis), randomised controlled trials (RCTs), cohort studies and case controlled studies comparing six interventions comprising different combinations of long-acting bronchodilators and inhaled corticosteroids in unison or on their own. The primary outcome is the reduction in moderate-to-severe exacerbations. Secondary outcomes include: lung function, quality of life, mortality and other adverse events. Titles and abstracts will screened by the primary researcher. A second reviewer will repeat this on a proportion of records. The Population, Intervention, Comparator, Outcomes and Study framework will be used for data extraction. A network meta-analyses of outcomes from RCTs and real-world evidence will be integrated if feasible. The $95 \%$ credible interval will be used to assess the statistical significance of each summary effect. Ranking of interventions will be based on their surface under cumulative ranking area.

Ethics and dissemination COPD exacerbations are burdensome to patients. We aim to report results that provide clinicians with a more informed choice of which inhaled therapy combinations are best to reduce exacerbations, improve disease burden and reduce lung function and exercise capacity decline, compared with the potential harms, in certain populations with COPD. PROSPERO registration number CRD42018088013.

\section{BACKGROUND}

\section{Rationale}

Chronic obstructive pulmonary disease (COPD) affects around 174 million persons worldwide. ${ }^{1}$ The most common cause is

\section{Strengths and limitations of this study}

- The planned systematic literature review and network meta-analysis will evaluate both randomised controlled trials and real-world evidence in order to reflect the current best evidence-based practice and increase the application to a potentially heterogeneous group of patients with COPD.

- A ranking system of all inhaled treatment comparisons will enable clinicians to judge each intervention on their evidence of clinical benefit compared with their potential harms.

- It may not be possible to combine both randomised controlled trials and other study designs in a single network meta-analysis.

smoking, and patients exhibit airflow obstruction that is not fully reversible. The disease is progressive, with declining lung function and a worsening of symptoms. Most troublesome are acute exacerbations manifested as a sudden worsening of symptoms, for example, severe coughing, shortness of breath and chest congestion, requiring urgent treatment and possibly hospitalisation. While smoking cessation remains the most effective intervention, the rate of exacerbation can be reduced by regular medication such as combination of long-acting beta-adrenoceptor agonists (LABAs), ${ }^{2}$ and inhaled corticosteroids (ICS) ${ }^{34}$ or long-acting muscarinic antagonists (LAMAs). ${ }^{5}$

COPD treatment guidelines are largely informed by randomised controlled trial (RCT) results, ${ }^{6}$ but we do not know if these findings apply to large patient populations not studied in trials. While RCTs will continue to be the gold standard for assessing the efficacy of medical interventions, they are expensive to conduct, and for practical and ethical 
reasons usually involve testing treatments in patient populations and within contexts which are sometimes very different from real life. In particular, RCT participants with COPD usually have no comorbidities and are otherwise healthy. Whereas often the very patients who are most commonly seen in a COPD outpatient clinic are those that would be excluded from clinical trials due to their comorbidities. As patient populations with chronic diseases such as COPD become more complex, the studies used to generate clinical evidence must reflect this.

Evidence of medication effects in routine care is vital for understanding the balance of treatment risks and benefits. RCTs have unique strengths, but results from trials are not always a good guide to the effects of drugs in routine clinical practice. ${ }^{7}$ National drug licensing authorities are now demanding better real-world evidence on which to make decisions and have introduced legislation mandating studies of both effectiveness and risk to be conducted in routine clinical care rather than the narrow and optimal confines of most randomised trials. ${ }^{89}$ While the conduct of observational studies to investigate possible drug harms is well established, the use of these studies to estimate treatment effectiveness is in its infancy. Issues of treatment channelling and indication bias mean that measuring the intended benefit of a treatment is beset with difficulties. While others have demonstrated it can be done in certain circumstances, we need more certainty about the methodology as it is applied in each disease area, since the issues of bias are likely to vary considerably. ${ }^{9}$

There is increasing evidence that dual long-acting bronchodilator therapy (LABA+LAMA) is more clinically beneficial compared with monotherapy or placebo ${ }^{10}$; however, there is also evidence that adding an ICS to LABA and LABA as triple therapy is better at reducing exacerbations and maintaining lung function. ${ }^{11}$ Furthermore, new treatments for reducing exacerbation frequency have become more evidenced based in recent years with commonly inhaled therapies (LABAs, LAMAs, ICS and combination devices) being prescribed alongside other treatments such as mucolytics (carbocysteine, $N$-acetylcysteine) ${ }^{12}$ antibiotics (macrolides) and inhaled colomycin ${ }^{13} 14$ and phosodiesterase- 4 inhibitors such as roflumilast. ${ }^{15}$

Until recently, dual combination therapy (LABA+ICS) was the recommended treatment for highly symptomatic COPD patients at risk of exacerbations or severe airflow limitation. ${ }^{16}$ However, in the Global Initiative for Chronic Obstructive Lung Disease (GOLD) guidelines $(2017)^{17}$ triple therapy (combined LABA+LAMA+ICS) is only recommended for patients categorised as severe. Given this changing landscape, and the future launch of combination triple therapies, it is important to identify and synthesise the available evidence from clinical trials and observational studies.

\section{Objectives}

This systematic literature review will assess clinical benefits and adverse events from maintenance treatment combinations in COPD described by RCTs and realworld evidence (RWE) studies. A network meta-analyses of outcomes from RCTs and RWE will be integrated if feasible.

The primary research question is: What are the clinical benefits of maintenance treatment combinations in COPD in terms of reducing exacerbations?

Secondary research questions include:

1. What is the long-term effectiveness of treatment combinations on improving lung function as measured by forced expiratory volume in $1 \mathrm{~s}\left(\mathrm{FEV}_{1}\right)$ ?

2. What is the long-term effectiveness of treatment combinations in improving patient-reported outcomes of respiratory-related quality of life, as measured by the St Georges Respiratory Questionnaire or the COPD Assessment Test?

3. What are the harms of each treatment combination including mortality, cardiovascular events, pneumonia, diabetes and bone fractures?

\section{METHODS}

This protocol has been prepared using the Preferred Reporting Items for Systematic Reviews and Meta-Analyses Protocols guidelines. ${ }^{18}$

\section{Eligibility criteria}

\section{Study design/characteristics}

Target studies will include RCTs, cohort studies and casecontrol studies comparing each intervention of interest to any of the possible comparators and looking at any of the possible outcomes (see below and table 1). We will also use summary papers, systematic reviews and other analyses to source relevant primary papers.

\section{Participants}

Adults diagnosed with COPD defined by a fixed post-bronchodilator ratio of forced expiratory volume in $1 \mathrm{~s}$ to forced vital capacity $\left(\mathrm{FEV}_{1} / \mathrm{FVC}\right)$ of less than 0.70 . Other measures of impaired lung function, such as lower limit of normal of $\mathrm{FEV}_{1} / \mathrm{FVC}$ ratio, will not be used to judge diagnosis in this review. The population will be clinically stable at the time of recruitment, defined as not having been treated for an exacerbation in the prior 6 weeks.

\section{Interventions}

There are six interventions of interest: (1) LABA, (2) LAMA, (3) LABA+LAMA, (4) ICS, (5) LABA+ICS and (6) LABA+LAMA + ICS.

\section{Comparators}

Each intervention may be compared against each other intervention or a placebo (table 1).

\section{Outcome}

The primary outcome of interest is the number of moderate-to-severe exacerbations experienced by patients following intervention. Secondary outcomes include $\mathrm{FEV}_{1}$ change from baseline, mortality (all-cause and 
Table 1 Summary of participants, intervention, comparators and outcomes (PICO) criteria

Participants: Adults diagnosed with COPD defined by a fixed postbronchodilator $\mathrm{FEV}_{1} / \mathrm{FVC}$ ratio of less than 0.70 .

\begin{tabular}{|c|c|c|}
\hline Interventions & Comparators & Outcomes \\
\hline 1. $\angle A B A$ & $\begin{array}{ll}\text { a. } & \text { LAMA } \\
\text { b. } \\
\text { c. ICBA+LAMA } \\
\text { d. LABA+ICS } \\
\text { e. LABA+LAMA + ICS } \\
\text { f. Placebo }\end{array}$ & $\begin{array}{l}\text { 1. Number of moderate-to- } \\
\text { severe exacerbations } \\
\text { 2. FEV change from } \\
\text { baseline } \\
\text { 3. Mortality } \\
\text { 4. Respiratory-related }\end{array}$ \\
\hline 2. LAMA & $\begin{array}{l}\text { a. } L A B A \\
\text { b. } L A B A+\angle A M A \\
\text { c. ICS } \\
\text { d. LABA+ICS } \\
\text { e. LABA+LAMA + ICS } \\
\text { f. Placebo }\end{array}$ & $\begin{array}{l}\text { quality of life (SGRQ, } \\
\text { CAT) } \\
\text { 5. Number of adverse and } \\
\text { serious adverse events: } \\
\text { a. Cardiovascular } \\
\text { events } \\
\text { b. Pneumonia }\end{array}$ \\
\hline 3. $\angle A B A+L A M A$ & $\begin{array}{l}\text { a. } L A B A \\
\text { b. } L A M A \\
\text { c. ICS } \\
\text { d. LABA+ICS } \\
\text { e. LABA+LAMA + ICS } \\
\text { f. Placebo }\end{array}$ & $\begin{array}{l}\text { c. Diabetes } \\
\text { d. Bone fractures }\end{array}$ \\
\hline 4. ICS & $\begin{array}{l}\text { a. } L A B A \\
\text { b. } L A M A \\
\text { c. LABA+LAMA } \\
\text { d. LABA+ICS } \\
\text { e. LABA+LAMA + ICS } \\
\text { f. Placebo }\end{array}$ & \\
\hline 5. $L A B A+I C S$ & $\begin{array}{l}\text { a. } \text { LABA } \\
\text { b. LAMA } \\
\text { c. LABA+LAMA } \\
\text { d. ICS } \\
\text { e. LABA+LAMA + ICS } \\
\text { f. Placebo }\end{array}$ & \\
\hline $\begin{array}{l}\text { 6. LABA+LAMA } \\
+ \text { ICS }\end{array}$ & $\begin{array}{ll}\text { a. } & \text { LABA } \\
\text { b. } & \text { LAMA } \\
\text { c. } & \text { LABA+LAMA } \\
\text { d. ICS } \\
\text { e. LABA+ICS } \\
\text { f. Placebo }\end{array}$ & \\
\hline
\end{tabular}

COPD, chronic obstructive pulmonary disease; CAT, COPD Assessment Tool; FEV ${ }_{1}$, forced expiratory volume in 1s; FVC, forced vital capacity; ICS, inhaled corticosteroids; LABA, long-acting beta-adrenoceptor agonist; LAMA, long-acting muscarinic antagonists; SGRQ, St Georges Respiratory Questionnaire.

respiratory-related), respiratory-related quality of life as measured by the St Georges Respiratory Questionnaire (SGRQ) or the COPD Assessment Tool (CAT) and the number of adverse and serious adverse events following intervention including cardiovascular events, pneumonia, diabetes and bone fractures.

\section{Information sources}

MEDLINE (Ovid interface, 1980 onwards) and Embase (Ovid interface, 1980 onwards) will be searched for potentially relevant articles using pre-defined search strategies. The International Prospective Register of Systematic Reviews (PROSPERO) will be searched periodically for relevant ongoing and completed systematic reviews. A manual search of the reference lists of all included studies will be conducted to check for other possibly relevant articles.

Manual searches will also be conducted to capture data not reported in the main publications and data from recent studies not yet published. Manual searches are anticipated to include conferences of interest and proceedings published from 2015 onwards, based on the assumption that trials are usually published within 2 to 3 years following presentation to a conference. In addition, we will search agencies' websites (National Institute for Health and Care Excellence etc) and clinical trial registry clinicaltrials.gov.

Studies will be excluded if they investigate only single short-acting inhaled therapy versus placebo. Additionally, studies will be excluded if they exclude patients on the basis of comorbidities that are unlikely to be affected by the trialled drug.

\section{Search strategy}

The list of proposed search terms (table 2) will be reviewed by the primary and secondary reviewer and research group lead. The final search strategy will be checked for accuracy by the guarantor using the Peer Review of Electronic Search Strategies (PRESS) checklist. ${ }^{19}$ The search strategy will be developed in the first instance for MEDLINE and then adapted for use with Embase. Search filters limiting to studies published in 1980 onwards, studies published in English and studies in humans will be used. The disease terms, inhaler terms and outcome searches will be combined using the Boolean logic operator AND, while different choices of terms within these categories using will be combined using OR. The literature review searches will be updated at the end of the process.

\section{Study records}

\section{Data management}

Literature search results will be uploaded and stored in EndNote (X8) and duplicates will be removed.

\section{Selection process}

The selection of studies for inclusion in the review will be conducted in two stages. First, titles and abstracts of all records identified by the database searches will screened by the primary researcher against the pre-defined eligibility criteria in order to identify a subset of potentially relevant studies. A second reviewer will repeat this exercise on a proportion of records and any discrepancies resolved by discussion. To reduce the risk of missing potentially relevant studies, a deliberately lenient approach will be adopted for this first level of screening by title and abstract.

We will obtain full reports for all titles that appear to meet our eligibility criteria, or where there is uncertainty. Full text screening will be also conducted by the primary researcher. Supplementary material will be consulted if the information provided in the main published article is insufficient to assess whether or not the inclusion criteria are met. Any discrepancies will be resolved by discussion and/or consultation with a third reviewer with specific expertise in COPD. A record will be kept of the reasons 


\section{Table 2 Search terms (provisional)

\begin{tabular}{ll}
\hline Concept & Search Terms \\
\hline $\begin{array}{l}\text { Chronic obstructive } \\
\text { pulmonary disease }\end{array}$ & $\begin{array}{l}\text { MeSH terms: exp Pulmonary Disease, } \\
\text { Chronic Obstructive/ } \\
\text { Free text terms: COPD, chronic } \\
\text { obstructive pulmonary disease }\end{array}$ \\
LABA & $\begin{array}{l}\text { Free text terms: arformoterol, } \\
\text { formoterol, indacaterol, olodaterol, } \\
\text { salmeterol, foradi, vilanterol, onbrez, } \\
\text { oxis, salmeterol, tulobuterol, vilanterol, } \\
\text { bambuterol, bambec, atimos } \\
\text { modulate, striverdi respimat, serevent, } \\
\text { relvar ellipta }\end{array}$
\end{tabular}

\begin{tabular}{|c|c|}
\hline LAMA & $\begin{array}{l}\text { Free text terms: aclidinium bromide, } \\
\text { glycopyrronium bromide, tiotropium, } \\
\text { umeclidinium, anoro ellipta, eklira, } \\
\text { seebri, Spiriva, incruse, antimuscarinic } \\
\text { bronchodilators, glycopyrrolate }\end{array}$ \\
\hline ICS & $\begin{array}{l}\text { Free text terms: beclomethasone, } \\
\text { budesonide, fluticasone furoate, } \\
\text { fluticasone propionate, fluticasone, } \\
\text { mometasone furoate, alvesco, } \\
\text { betamethasone, ciclesonide, flixotide, } \\
\text { pulmicort, becloforte, becotide, relvar } \\
\text { ellipta }\end{array}$ \\
\hline
\end{tabular}

\begin{tabular}{ll} 
Exacerbation & $\begin{array}{l}\text { MeSH terms: exp respiratory tract } \\
\text { infections/ } \\
\text { Free text terms: exacerbation, } \\
\text { AECOPD, lower respiratory tract } \\
\text { infection, LRTI }\end{array}$ \\
& Forced expiratory volume in 1s (FEV $\left.{ }_{1}\right)$ \\
\hline FEV ${ }_{1}$ & MeSH terms: exp Mortality/exp death/ \\
Mortality & Free text terms: mortality, death \\
Quality of life & $\begin{array}{l}\text { MeSH terms: exp quality of life/ } \\
\text { Free text terms: quality of life, St } \\
\text { Georges Respiratory Questionnaire } \\
\text { (SGRQ), COPD Assessment Test (CAT) }\end{array}$ \\
Myocardial & $\begin{array}{l}\text { MeSH terms: exp myocardial } \\
\text { infarction/ } \\
\text { Infarction }\end{array}$ \\
& $\begin{array}{l}\text { Free text terms: myocardial infarction } \\
\text { (MI) } \\
\text { MeSH terms: exp stroke/ } \\
\text { Free text terms: stroke }\end{array}$ \\
\hline
\end{tabular}

\begin{tabular}{ll} 
Arrhythmias & $\begin{array}{l}\text { MeSH terms: exp arrhythmias, cardiac/ } \\
\text { exp atrial fibrillation/ } \\
\text { Free text terms: arrhythmia, atrial } \\
\text { fibrillation }\end{array}$ \\
Angina & $\begin{array}{l}\text { MeSH terms: exp angina, unstable/ } \\
\text { exp angina pectoris } \\
\text { Free text terms: unstable angina, } \\
\text { angina pectoris }\end{array}$ \\
Pneumonia & $\begin{array}{l}\text { MeSH terms: exp pneumonia/ } \\
\text { Free text terms: pneumonia }\end{array}$ \\
Type-2 Diabetes & $\begin{array}{l}\text { MeSH terms: exp diabetes mellitus, } \\
\text { type 2/ } \\
\text { Free text terms: type 2 diabetes } \\
\text { (T2DM) }\end{array}$ \\
\hline
\end{tabular}

Continued
Table 2 Continued

\begin{tabular}{ll}
\hline Concept & Search Terms \\
\hline Fractures & $\begin{array}{l}\text { MeSH terms: exp osteoporotic } \\
\text { fractures/exp fractures, bone } \\
\end{array}$ \\
& $\begin{array}{l}\text { Free text terms: osteoporotic fractures, } \\
\text { bone fractures }\end{array}$ \\
\hline
\end{tabular}

ICS, inhaled corticosteroids; LABA, long-acting beta-adrenoceptor agonist; LAMA, long-acting muscarinic antagonists.

for rejection of articles during the full-text screening process.

\section{Data extraction}

Information will be extracted from all selected studies using a pre-specified data extraction form. The form will be piloted on the first six selected studies and refined as necessary. Online supplementary material will be consulted and/or authors contacted if the information provided in the original published articles is insufficient to complete the extraction.

As with screening, data extraction will be carried out in duplicate on a proportion of selected records to reduce the risk of errors and introducing bias. Similarly, any discrepancies will be resolved by discussion and/or consultation with the third reviewer.

\section{Data items}

We will use the PICOs (Population, Intervention, Comparator, Outcomes and Study characteristics) framework to systematise our data extraction. Within each of these five domains, information will be recorded on the following items:

- The study characteristics including setting, design, period of study, aims and objectives.

- The population including characteristics (including size, sex and age distribution, ethnicity, country), recruitment and sampling methods, inclusion/exclusion criteria.

- The intervention status definition and identification, number of exposed subjects, any exclusions.

- The comparators' identification and definition of unexposed individuals, number of unexposed subjects, any exclusions.

- The outcomes definitions and identification of primary (exacerbation) and secondary outcomes ( $\mathrm{FEV}_{1}$ change from baseline, mortality, respiratory-related quality of life, adverse events), number of subjects, any exclusions, length of follow-up.

\section{Outcomes and prioritisation}

The following list of endpoints is anticipated; to protect against study selection bias for the length of studies, we anticipate splitting our analyses of exacerbations and lung function by length of study.

1. Number of moderate-to-severe exacerbations

a. Short-term treatment $(<20$ weeks)

b. Long-term treatment ( $\geq 20$ weeks) 
2. $\mathrm{FEV}_{1}$ change from baseline

a. Short-term treatment $(<12$ weeks)

b. Long-term treatment ( $\geq 12$ weeks)

3. Mortality

4. Respiratory-related quality of life (SGRQ, CAT)

5. Number of adverse and serious adverse events including:

a. Cardiovascular events

b. Pneumonia

c. Diabetes

d. Bone fractures

\section{Risk of bias assessment}

The primary reviewer will assess the risk of bias of each study included using the seven-item Cochrane Risk of Bias Tool. $^{20}$ The Critical Appraisal Skills Programme ${ }^{21}$ quality assessment tools will be used to assess the quality of observational studies. Risk of bias assessment will be conducted independently by a second reviewer on a quarter of the selected studies and disagreements will be resolved in the first instance by discussion and then through consultation with a third reviewer. On completion of this process, the remaining studies will be assessed for the risk of bias by one reviewer. The Alternative Regression Test ${ }^{22}$ will be applied to evidence to test for publication bias.

\section{Data synthesis}

Three key aspects in the assessment of feasibility of network meta-analysis (NMA) (RCTs, RWE) will be examined:

1. The validity of an NMA of RCTs may be compromised by looking at: (1) clinical heterogeneity, defined as the extent of confounding bias due to an RCT's similarity and consistency violations and (2) methodological heterogeneity arising from the quality of the individual RCTs that make up the evidence network. The assessment of clinical heterogeneity will be based on evaluation of the following data elements extracted from the RCTs:

a. The study design, including the quality of methods employed.

b. The admissibility criteria in terms of confounding factors (including subgroups of interest).

c. The measurement and reporting of outcomes (eg, definitions of progression and time points, testing assumptions for underlying distributions).

d. The comparators (common reference intervention) and dosages, schedules and routes of administration.

2. The validity of an NMA of RWE by looking at the similarity of patients populations across studies, similarity of treatments and differences in outcomes.

3. The use of complex statistical methods to control for confounding such as propensity score, disease risk score, and stabilised inverse probability weighting method.

Descriptive statistics will be conducted to describe the following:
- The main characteristics of the study populations at baseline (eg, age, sex, proportion of smokers, disease category as determined by the GOLD standard (A,B,C,D), $\mathrm{FEV}_{1}$ status, etc.)

- Study designs (eg, details specific to RCT and RWE).

- Whenever possible, graphs showing the characteristics of studies by treatment or study will be developed. These findings will be used as a basis to identify potential sources of heterogeneity and to detect any potential outlier.

The problem of bias due to systematically differing entry criteria is not something we can pre-requisitely prevent; however, should there be large differences in population characteristics, we will conduct appropriate subgroup analyses.

\section{Network meta-analysis}

This NMA will incorporate the scenarios of both indirect and mixed treatment comparisons. The NMA will initially trial in feasibility design whether RCTs can be compared solely with other RCT designs of similar methodology or whether other types of study design such as observational studies can be included in direct comparison. Heterogeneity and inconsistency will be tested using the Wald test for inconsistency.

Potential differences across studies may include:

- The treatment dose/schedule or outcome definitions (exacerbation description).

- The distribution of each study and patient characteristics.

- Baseline risks associated with each treatment.

- Observed treatment effects.

In order to account for these differences, methods of meta-regression, sub-group and sensitivity analysis may be employed.

Should it not be possible to compare all study designs in a single NMA to show best-efficacy ranking of interventions, then separate NMAs will be performed for RCTs and the other study designs. The geometry of the network for each outcome measure will be presented in a network diagram of treatment nodes of variable sizes, comparison lines of variable thickness and loops to identify treatments with both direct and indirect comparisons. ${ }^{23}$

We plan to provide an overall combined ranking of interventions depending on their effect on exacerbation reduction compared with the combined weight of harm per intervention. Each harm will be weighted based on the severity of any harm reported as a result of using each intervention in each study. The study with best effect and least harm will be ranked first.

\section{Statistical analysis}

Due to the nature of this NMA, it is assumed that a random effects meta-analysis model will be chosen for the primary outcome of rate of exacerbation and continuous outcomes of health related quality of life and $\mathrm{FEV}_{1}$. To estimate patient years for exacerbations, deaths and adverse events we plan to use methods previously 
reported. ${ }^{24}$ For change in continuous outcomes, that is, $\mathrm{FEV}_{1}$ and SGRQ, we will either use the reported change or calculate the change from baseline based on the reported values. We will calculate the SD of change from the reported standard errors or confidence intervals. The primary outcome of risk of exacerbation will be reported as rate ratio and other continuous outcome measures will be reported as mean differences assuming normal distribution of data. The $95 \%$ CI for each summary statistics will be used to assess the statistical significance of each summary effect. The ranking of interventions will be based on their surface under cumulative ranking area, allowing clinicians to make treatment decisions based on relative effectiveness and risk of harm. ${ }^{25}$ Statistical analysis will be performed using Stata (StataCorp LP, College Station, TX, USA) and R (R Foundation for Statistical Computing, Vienna, Austria).

\section{Confidence in cumulative evidence}

The quality of evidence for our primary and secondary research questions will be assessed using those domains of the Grading of Recommendations, Assessment, Development, and Evaluations (GRADE) guidelines that are pertinent to observational studies, notably those relating to methodological flaws within the component studies and the consistency of results across different studies. Additional domains may be considered if deemed appropriate. The strength of evidence will be judged as 'high' (further research is unlikely to change our conclusion), 'moderate' (further research is likely to alter our conclusion) or 'low' (further studies are required to answer the research question with a high degree of confidence/ increase confidence).

\section{Patient involvement}

No patients were involved in the design or analysis of this study.

\section{ETHICS AND DISSEMINATION}

Ethical review is not required as this study is a systematic review. It is our intention to submit the results of our review for peer-reviewed publication and to present our findings at national and international meetings and conferences.

Contributors AL and ELA drafted the protocol and developed the inclusion/ exclusion criteria, the risk of bias assessment tool, the data extraction form and searching with guidance from JKQ. AL registered and updated the review on PROSPERO. AL, ELA, JP, RT, HV and JKQ contributed to the initial scoping discussions of the research, participated in decisions regarding the risk of bias assessment strategies and contributed to drafting the manuscript. JP provided guidance and contributed to the statistical plan. JKQ, AL and ELA participated in the protocol submission. JKQ is the guarantor of the review.

Funding Boehringer Ingelheim Ltd. Bracknell, UK. Writing and research was funded by Boehringer Ingelheim Ltd; however, the publication of study results is not contingent on the sponsor's approval or censorship of the manuscript.

Competing interests AL was funded by Boehringer Ingelheim Ltd to perform this systematic review and network meta-analysis. ELA has nothing to declare. JP has nothing to declare. RT and HV are employees of Boehringer Ingelheim Ltd. JKQ's research group has received funding from The Health Foundation, MRC, Wellcome
Trust, BLF, GSK, Insmed, AZ and Bayer for other projects, none of which relate to this work. BI have funded this work. JKQ has received funds from AZ, GSK, Chiesi, Teva and BI for Advisory board participation or travel.

Patient consent for publication Not required.

Provenance and peer review Not commissioned; externally peer reviewed.

Open access This is an open access article distributed in accordance with the Creative Commons Attribution Non Commercial (CC BY-NC 4.0) license, which permits others to distribute, remix, adapt, build upon this work non-commercially, and license their derivative works on different terms, provided the original work is properly cited, appropriate credit is given, any changes made indicated, and the use is non-commercial. See: http://creativecommons.org/licenses/by-nc/4.0/.

\section{REFERENCES}

1. Rabe KF, Watz H. Chronic obstructive pulmonary disease. Lancet 2017;389:1931-40

2. Appleton S, Poole P, Smith B, et al. Long-acting beta2-agonists for poorly reversible chronic obstructive pulmonary disease. Cochrane Database Syst Rev 2006;3:CD001104.

3. Zhong N, Zheng J, Wen F, et al. Efficacy and safety of budesonide/ formoterol via a dry powder inhaler in Chinese patients with chronic obstructive pulmonary disease. Curr Med Res Opin 2012;28:257-65.

4. Zheng JP, Yang L, Wu YM, et al. The efficacy and safety of combination salmeterol (50 microg)/fluticasone propionate (500 microg) inhalation twice daily via accuhaler in Chinese patients with COPD. Chest 2007;132:1756-63.

5. Karner C, Chong J, Poole P. Tiotropium versus placebo for chronic obstructive pulmonary disease. Cochrane Database Syst Rev 2014:CD009285.

6. Global initiative for chronic obstructive lung disease (GOLD), 2011.

7. van Staa TP, Leufkens HG, Zhang B, et al. A comparison of cost effectiveness using data from randomized trials or actual clinical practice: selective cox-2 inhibitors as an example. PLoS Med 2009;6:e1000194.

8. Beeh KM, Beier J. The short, the long and the "ultra-long": why duration of bronchodilator action matters in chronic obstructive pulmonary disease. Adv Ther 2010;27:150-9.

9. Smeeth L, Douglas I, Hall AJ, et al. Effect of statins on a wide range of health outcomes: a cohort study validated by comparison with randomized trials. Br J Clin Pharmacol 2009;67:99-109.

10. Rodrigo GJ, Price D, Anzueto A, et al. LABA/LAMA combinations versus LAMA monotherapy or LABA/ICS in COPD: a systematic review and meta-analysis. Int $J$ Chron Obstruct Pulmon Dis 2017;12:907-22.

11. Zhu Y, Zhang T, Li H, et al. Discovering the Relative Efficacy of Inhaled Medications for Chronic Obstructive Pulmonary Disease: Multiple Treatment Comparisons. Cell Physiol Biochem 2017;41:1532-46.

12. Zheng JP, Wen FQ, Bai CX, et al. Twice daily N-acetylcysteine 600 $\mathrm{mg}$ for exacerbations of chronic obstructive pulmonary disease (PANTHEON): a randomised, double-blind placebo-controlled trial. Lancet Respir Med 2014;2:187-94.

13. Albert RK, Connett J, Bailey WC, et al. Azithromycin for prevention of exacerbations of COPD. N Engl J Med 2011;365:689-98.

14. Bruguera-Avila N, Marin A, Garcia-Olive I, et al. Effectiveness of treatment with nebulized colistin in patients with COPD. Int J Chron Obstruct Pulmon Dis 2017;12:2909-15.

15. Calverley PM, Rabe KF, Goehring UM, et al. Roflumilast in symptomatic chronic obstructive pulmonary disease: two randomised clinical trials. Lancet 2009;374:685-94.

16. Excellence NIfHaC. Chronic obstructive pulmonary disease in over 16s: diagnosis and management, 2010.

17. GOLD. Global Strategy for the Diagnosis, Management and Prevention of COPD, Global Initiative for Chronic Obstructive Lung Disease (GOLD) 2017 [Webpage]. 2017 http://goldcopd.org/gold2017-global-strategy-diagnosis-management-prevention-copd/ (accessed 10 Jan 2018).

18. Shamseer L, Moher D, Clarke M, et al. Preferred reporting items for systematic review and meta-analysis protocols (PRISMA-P) 2015: elaboration and explanation. BMJ 2015;349:g7647.

19. McGowan J, Sampson M, Salzwedel DM, et al. PRESS Peer Review of Electronic Search Strategies: 2015 guideline statement. J Clin Epidemiol 2016;75:40-6.

20. Higgins JP, Altman DG, Gøtzsche PC, et al. The Cochrane Collaboration's tool for assessing risk of bias in randomised trials. BMJ 2011;343:d5928.

21. Program CAS. https://casp-uk.net/2018 
22. Peters JL, Sutton AJ, Jones DR, et al. Comparison of two methods to detect publication bias in meta-analysis. JAMA 2006;295:676-80.

23. Tonin FS, Rotta I, Mendes AM, et al. Network meta-analysis: a technique to gather evidence from direct and indirect comparisons. Pharm Pract 2017;15:943.
24. Loymans RJ, Gemperli A, Cohen J, et al. Comparative effectiveness of long term drug treatment strategies to prevent asthma exacerbations: network meta-analysis. BMJ 2014;348:g3009.

25. Mbuagbaw L, Rochwerg B, Jaeschke R, et al. Approaches to interpreting and choosing the best treatments in network metaanalyses. Syst Rev 2017;6:79. 